\title{
Cyclic partitions of complete uniform hypergraphs
}

\author{
Artur Szymański \\ szymanski@artgraph.eu
}

\author{
A. Paweł Wojda* \\ Faculty of Applied Mathemetics \\ AGH University of Science and Technology \\ Cracow, Poland \\ wojda@agh.edu.pl
}

Submitted: Jun 4, 2010; Accepted: Aug 5, 2010; Published: Sep 1, 2010

Mathematics Subject Classifications: 05C65

\begin{abstract}
By $K_{n}^{(k)}$ we denote the complete $k$-uniform hypergraph of order $n, 1 \leqslant k \leqslant n-1$, i.e. the hypergraph with the set $V_{n}=\{1,2, \ldots, n\}$ of vertices and the $\operatorname{set}\left(\begin{array}{c}V_{n} \\ k\end{array}\right)$ of edges. If there exists a permutation $\sigma$ of the set $V_{n}$ such that $\left\{E, \sigma(E), \ldots, \sigma^{q-1}(E)\right\}$ is a partition of the set $\left(\begin{array}{c}V_{n} \\ k\end{array}\right)$ then we call it cyclic $q$-partition of $K_{n}^{(k)}$ and $\sigma$ is said to be a $(q, k)$-complementing.

In the paper, for arbitrary integers $k, q$ and $n$, we give a necessary and sufficient condition for a permutation to be $(q, k)$-complementing permutation of $K_{n}^{(k)}$.

By $\tilde{K}_{n}$ we denote the hypergraph with the set of vertices $V_{n}$ and the set of edges $2^{V_{n}}-\left\{\emptyset, V_{n}\right\}$. If there is a permutation $\sigma$ of $V_{n}$ and a set $E \subset 2^{V_{n}}-\left\{\emptyset, V_{n}\right\}$ such that $\left\{E, \sigma(E), \ldots, \sigma^{p-1}(E)\right\}$ is a $p$-partition of $2^{V_{n}}-\left\{\emptyset, V_{n}\right\}$ then we call it a cyclic $p$-partition of $K_{n}$ and we say that $\sigma$ is $p$-complementing. We prove that $\tilde{K}_{n}$ has a cyclic $p$-partition if and only if $p$ is prime and $n$ is a power of $p$ (and $n>p$ ). Moreover, any $p$-complementing permutation is cyclic.
\end{abstract}

\section{Preliminaries and results}

Throughout the paper we will write $V_{n}=\{1, \ldots, n\}$. For a set $X$ we denote by $\left(\begin{array}{l}X \\ k\end{array}\right)$ the set of all $k$-subsets of $X$. A hypergraph $H=(V ; E)$ is said to be $k$-uniform if $E \subset\left(\begin{array}{l}V \\ k\end{array}\right)$ (the cardinality of any edge is equal to $k$ ). We shall always assume that the set of vertices $V$ of a hypergraph of order $n$ is equal to $V_{n}$. The complete $k$-uniform hypergraph of order $n$ is denoted by $K_{n}^{(k)}$, hence $K_{n}^{(k)}=\left(V_{n} ;\left(\begin{array}{c}V_{n} \\ k\end{array}\right)\right)$. Let $\sigma$ be a permutation of the set $V_{n}$, let $q$ be a positive integer, and let $E \subset\left(\begin{array}{c}V_{n} \\ k\end{array}\right)$. If $\left\{E, \sigma(E), \sigma^{2}(E), \ldots, \sigma^{q-1}(E)\right\}$ is a partition of $\left(\begin{array}{c}V_{n} \\ k\end{array}\right)$ we call it a cyclic $q$-partition and $\sigma$ is said to be $(q, k)$-complementing. It is

*The research of APW was partially sponsored by polish Ministry of Science and Higher Education. 
very easy to prove that then $\sigma^{q}(E)=E$. Write $E_{i}=\sigma^{i}(E)$ for $i=0, \ldots, q-1$. It follows easily that $\sigma^{t}\left(E_{i}\right)=E_{i+t}(\bmod q)$, for every integer $t$.

If there is a cyclic 2-partition $\{E, \sigma(E)\}$ of $K_{n}^{(k)}$, we say that the hypergraph $H=\left(V_{n} ; E\right)$ is self-complementary and every $(2, k)$-complementing permutation of $K_{n}^{(k)}$ is called self-complementing. In [16] we have given the characterization of selfcomplementing permutations which, as it turns out, is exactly Theorem 2 of this paper for $p=2, \alpha=1$. Self-complementary $k$-uniform hypergraphs generalize the self-complementary graphs defined in [13] and [14]. The vertex transitive self-complementary $k$-uniform hypergraphs are the subject of the paper [11] by Potǒcnik and Šajna. Gosselin gave an algorithm to construct some special self-complementary $k$-uniform hypergraphs in [3]. In [6] and [10] Knor, Potǒcnik and Šajna study the existence of regular self-complementary $k$-uniform hypergraphs.

The main result of this paper is a necessary and sufficient condition for a permutation $\sigma$ of $V_{n}$ to be $(q, k)$-complementing, where $q$ is a positive integer (Theorem 3 ). In Theorem 5 we characterize integers $n, k, \alpha$ and primes $p$ such that there exists a cyclic $p^{\alpha}$-partition of $K_{n}^{(k)}$.

Section 2 contains the proofs of Theorems 1, 2 and 3 given below. Section 3 is devoted to cyclic partitions of complete hypergraph $\tilde{K}_{n}=\left(V_{n} ; 2^{V_{n}}-\left\{\emptyset, V_{n}\right\}\right.$ ) (we call $\tilde{K}_{n}$ the general complete hypergraph of order $n$, to stress the distinction between complete uniform and complete hypergraphs).

Theorem 1 Let $n$ and $k$ be integers, $0<k<n$, let $p_{1}$ and $p_{2}$ be two relatively prime integers. A permutation $\sigma$ on the set $V_{n}$ is $\left(p_{1} p_{2}, k\right)$-complementing if and only if $\sigma$ is $a\left(p_{j}, k\right)$-complementing for $j=1,2$.

For integers $n$ and $d, d>0$, by $r(n, d)$ we denote the reminder when $n$ is divided by $d$. So we have $n \equiv r(n, d)(\bmod d)$.

For a positive integer $k$ by $C_{p}(k)$ we denote the maximum integer $c$ such that $k=p^{c} a$, where $a \in \mathbf{N}$ ( $\mathbf{N}$ stands for the sets of naturals, i.e. nonnegative integers). In other words, if $k=\sum_{i \geqslant 0} k_{i} p^{i}$, where $0 \leqslant k_{i}<p$ for every $i \in\{0,1, \ldots\}\left(k_{i}\right.$ are digits with respect to basis $p)$, then $C_{p}(k)=\min \left\{i: k_{i} \neq 0\right\}$. If $A$ is a finite set, we write $C_{p}(A)$ instead of $C_{p}(|A|)$, for short.

Theorem 2 Let $n, p, k$ and $\alpha$ be positive integers, such that $k<n$ and $p$ is prime. A permutation $\sigma$ of the set $V_{n}$ with orbits $O_{1}, \ldots, O_{m}$ is $\left(p^{\alpha}, k\right)$-complementing if and only if there is a non negative integer $l$ such that the following two conditions hold:

(i) $r\left(n, p^{l+\alpha}\right)<r\left(k, p^{l+1}\right)$, and

(ii) $\sum_{i: C_{p}\left(O_{i}\right)<l+\alpha}\left|O_{i}\right|=r\left(n, p^{l+\alpha}\right)$.

A condition slightly different from the above has been given (and proved by different method, independently) in [4]. 
Observe that for any permutation $\sigma$ of $V_{n}$ with orbits $O_{1}, \ldots, O_{m}$ we have $\sum_{i: C_{p}\left(O_{i}\right)<l+\alpha}\left|O_{i}\right| \equiv r\left(n, p^{l+\alpha}\right) \quad\left(\bmod \quad p^{l+\alpha}\right), \quad$ since $\quad \sum_{i=1}^{m}\left|O_{i}\right|=n$ and $\sum_{i: C_{p}\left(O_{i}\right) \geqslant l+\alpha}\left|O_{i}\right| \equiv 0\left(\bmod p^{l+\alpha}\right)$. Hence the condition (ii) of Theorem 2 could be written equivalently: $\sum_{i: C_{p}\left(O_{i}\right)<l+\alpha}\left|O_{i}\right| \leqslant r\left(n, p^{l+\alpha}\right)$.

Theorem 3 Let $q=p_{1}^{\alpha_{1}} p_{2}^{\alpha_{2}} \cdot \ldots \cdot p_{u}^{\alpha_{u}}$, where $p_{1}, \ldots, p_{u}$ are mutually different primes and $\alpha_{1}, \ldots, \alpha_{u}$ positive integers. A permutation $\sigma$ of the set $V_{n}$ with orbits $O_{1}, \ldots, O_{m}$ is $(q, k)$-complementing if and only if for every $j \in\{1, \ldots, u\}$ there is a positive integer $l_{j}$ such that the following two conditions hold:

(i) $r\left(n, p_{j}^{l_{j}+\alpha_{j}}\right)<r\left(k, p_{j}^{l_{j}+1}\right)$, and

(ii) $\sum_{i: C_{p_{j}}\left(O_{i}\right)<l_{j}+\alpha_{j}}\left|O_{i}\right|=r\left(n, p_{j}^{l_{j}+\alpha_{j}}\right)$.

For the special case of graphs (i.e. 2-uniform hypergraphs) Theorem 2 has been proved in [1].

One may apply Theorem 2 to check that every permutation of $V_{89}$ consisting of two orbits: one of cardinality 64 and the second of cardinality 25 is $(2,40)$-complementing. Every permutation of $V_{89}$ consisting of orbits $O_{1}$ and $O_{2}$ such that $\left|O_{1}\right|=81$ and $\left|O_{2}\right|=8$ is $(9,40)$-complementing. But it is easily seen (applying either Theorem 2 or Theorem 3 ) that there is no $(18,40)$-complementing permutation of $K_{89}^{(40)}$.

It has been proved in [15] that for given $n$ and $k$ there is a self-complementary $k$-uniform hypergraph of order $n$ if and only if $\left(\begin{array}{l}n \\ k\end{array}\right)$ is even (the corresponding result for graphs was proved first in [13] and [14], independently). The natural question arises: is it true, that if $\left(\begin{array}{l}n \\ k\end{array}\right)$ is divisible by $q$ then there is a cyclic $q$-partition of $K_{n}^{(k)}$ ?

The problem of divisibility of $\left(\begin{array}{l}n \\ k\end{array}\right)$ was considered in the literature many times, independently. The theorem we give below has been proved in 1852 by Kummer [8], it was rediscovered by Lucas [9] in 1878, then by Glaisher [2] in 1899 and finally, for $p=2$ and $\alpha=1$ only, by Kimball et al. [5] (for an elegant proof of Kummer's result and its connections with Last Fermat Theorem see [12]).

Theorem 4 (Kummer) Let $p$ be a prime and let $\left(n_{i}\right)$ and $\left(k_{i}\right)$ denote the sequences of digits of $n$ and $k$ in base $p$, so that $n=\sum_{i \geqslant 0} n_{i} p^{i}$ and $k=\sum_{i \geqslant 0} k_{i} p^{i}\left(0 \leqslant n_{i}, k_{i} \leqslant p-1\right.$ for every $i) . C_{p}\left(\left(\begin{array}{l}n \\ k\end{array}\right)\right)$ is equal to the number of indices $i$ such that either $k_{i}>n_{i}$, or there exists an index $j<i$ with $k_{j}>n_{j}$ and $k_{j+1}=n_{j+1}, \ldots, k_{i}=n_{i}$.

Let $p$ be a prime integer, $0<k<n, k=\sum_{i \geqslant 0} k_{i} p^{i}, n=\sum_{i \geqslant 0} n_{i} p^{i}$, where $k_{i}$ and $n_{i}$ are digits with respect to the basis $p$. Note that, by Theorem 2 , if there is a cyclic $p^{\alpha}$-partition of $K_{n}^{(k)}$ then there are integers $l$ and $m, 0 \leqslant m \leqslant l$, such that $n_{m}<k_{m}$, and $n_{l+\alpha-1}=n_{l+\alpha-2}=\ldots=n_{l+1}=0$ (if $\alpha>1$ ), and $n_{i}=k_{i}$ for $m<i \leqslant l$ (if $m<l$ ).

Conversely, if for indices $l$ and $m$ we have $n_{l+\alpha-1}=n_{l+\alpha-2}=\ldots=n_{l+1}=0$ (for $\alpha>1$ ), $n_{l}=k_{l}, n_{l-1}=k_{l-1}, \ldots, n_{m+1}=k_{m+1}$ (if $m<l$ ), and $n_{m}<k_{m}$, then any permutation of $V_{n}$ 
which has two orbits $O_{1}$ and $O_{2}$ such that $\left|O_{1}\right|=\sum_{i \geqslant l+\alpha} n_{i} p^{i}$ and $\left|O_{2}\right|=\sum_{i=0}^{l+\alpha-1} n_{i} p^{i}=$ $\sum_{i=0}^{l} n_{i} p^{i}$ is, by Theorem 2, $\left(p^{\alpha}, k\right)$-complementing. We are thus led to the following corollary of Theorem 2 .

Theorem 5 Let $n, k, p$ and $\alpha$ be positive integers such that $k<n$ and $p$ is prime. Suppose that $k=\sum_{i \geqslant 0} k_{i} p^{i}, n=\sum_{i \geqslant 0} n_{i} p^{i}$, where $k_{i}$ and $n_{i}$ are digits with respect to the basis $p$.

The complete $k$-uniform hypergraph $K_{n}^{(k)}$ has a cyclic $p^{\alpha}$-partition if and only if there exist nonnegative integers $l$ and $m, m \leqslant l$, such that $n_{m}<k_{m}, n_{i}=k_{i}$ for $m<i \leqslant l$, and $n_{l+1}=n_{l+2}=\ldots=n_{l+\alpha-1}=0$ (if $\left.\alpha>1\right)$.

It is clear that for $\alpha>1$ it may happen that $n, k$ and a prime $p$ satisfy the assumption of Theorem 4, but violate the condition (i) of Theorem 2. Hence, in general, it is not true that if $p^{\alpha}$ divides $\left(\begin{array}{l}n \\ k\end{array}\right)$ then there is a cyclic $p^{\alpha}$-partition of $K_{n}^{(k)}$. However, it is very easy to observe that Theorem 4 and Theorem 5 imply the following.

Corollary 6 Let $n, k$ and $p$ be positive integers such that $k<n$ and $p$ is prime. The complete $k$-uniform hypergraph $K_{n}^{(k)}$ has a cyclic p-partition if and only if $p \mid\left(\begin{array}{l}n \\ k\end{array}\right)$.

The problem whether for positive $n, k$ and $q$ there is a cyclic $q$-partition of $K_{n}^{(k)}$ is in general open (unless $q$ is a power of a prime).

\section{Proofs}

\subsection{Lemmas}

Lemma 1 Let $k, n, q$ be positive integers, $k<n$. A permutation $\sigma$ of the set $V_{n}$ is $(q, k)$-complementing if and only if $\sigma^{s}(e) \neq e$ for any subset $e \subset V_{n}$ of cardinality $k$ and $s \not \equiv 0(\bmod q)$.

Proof. If $\sigma$ is $(q, k)$-complementing, then there is a partition $E_{0} \cup \ldots \cup E_{q-1}$ of $\left(\begin{array}{c}V_{n} \\ k\end{array}\right)$ such that $E_{i}=\sigma\left(E_{i-1}\right)$ for $i=0, \ldots, q-1$ (considered $\left.\bmod q\right)$. Since the sets $E_{0}, \ldots, E_{q-1}$ are mutually disjoint, for every $e \in\left(\begin{array}{c}V_{n} \\ k\end{array}\right)$ if $\sigma^{s}(e)=e$ then $s \equiv 0(\bmod q)$.

Let us now sppose that $\sigma$ is a permutation of $V_{n}$ such that $\sigma^{s}(e) \neq e$ for $s \not \equiv 0$ $(\bmod q)$. We may apply the following simple algorithm of coloring the edges of $K_{n}^{(k)}$ with $q$ colors. Suppose that an edge $e \in\left(\begin{array}{c}V_{n} \\ k\end{array}\right)$ is not yet colored. We color $e$ with arbitrary color $i_{0} \in\{0,1, \ldots, q-1\}$ and for every $l$ we color $\sigma^{l}(e)$ with the color $i_{0}+l(\bmod q)$. When all the edges are colored, denote by $E_{i}$ the set of edges colored with the color $i$. It is clear that $E_{0} \cup \ldots \cup E_{q-1}$ is a partition of $\left(\begin{array}{c}V_{n} \\ k\end{array}\right)$ and that $\sigma\left(E_{i-1}\right)=E_{i}$ for $i=0,1, \ldots, q-1$.

Note that by the algorithm given in the proof of Lemma 1 we may obtain all cyclic $p$-partitions of $K_{n}^{(k)}$ generated by $\sigma$. 
The proof of Theorem 1 follows immediately by Lemma 1 and the fact that for relatively prime integers $p_{1}$ and $p_{2}$ we have $l \equiv 0\left(\bmod p_{1} p_{2}\right)$ if and only if $l \equiv 0\left(\bmod p_{1}\right)$ and $l \equiv p_{2}\left(\bmod p_{2}\right)$.

Lemma 2 Let $n, k, p$ and $\alpha$ be positive integers such that $k<n$ and $p$ is prime. The cyclic permutation $\sigma=(1,2, \ldots, n)$ is $\left(p^{\alpha}, k\right)$-complementig if and only if $C_{p}(n) \geqslant C_{p}(k)+\alpha$.

Proof. Assume first that $C_{p}(n)-C_{p}(k) \geqslant \alpha$. We shall prove that then the permutation $\sigma=(1,2, \ldots, n)$ is $\left(p^{\alpha}, k\right)$-complementing.

Observe that for any postive integer $s$ every orbit of the permutation $\sigma^{s}$ has the same cardinality.

By Lemma 1 it is sufficient to prove that for any edge $e \in\left(\begin{array}{c}V_{n} \\ k\end{array}\right)$ if $\sigma^{s}(e)=e$ then $s \equiv 0$ $\left(\bmod p^{\alpha}\right)$. So let us suppose that $\sigma^{s}(e)=e$, write $\tau=\sigma^{s}$ and denote by $\beta$ the cardinality of any orbit of $\tau$. Note that $\tau^{\beta}=i d_{V_{n}}$ (where $i d_{V_{n}}$ is the identity of the set $V_{n}$ ).

For every vertex $v \in e$ we have clearly $\tau(v) \in e$, hence every orbit of $\tau$ containing a vertex of $e$ is contained in $e$. Therefore $\beta \mid k$. So there is an integer $\gamma$ such that $k=\beta \gamma$. We have $\tau^{k}=\left(\tau^{\beta}\right)^{\gamma}=i d_{V_{n}}$, hence $\sigma^{s k}=i d_{V_{n}}$ and therefore $s k \equiv 0(\bmod n)$. This means that there is an integer $\delta$ such that $s k=\delta n$, so $s p^{C_{p}(k)} k^{\prime}=\delta p^{C_{p}(n)} n^{\prime}$ where $p \nmid k^{\prime}$ and $p \nmid n^{\prime}$. Since $C_{p}(n)-C_{p}(k) \geqslant \alpha$ the equality $s k^{\prime}=\delta p^{\alpha} p^{C_{p}(n)-C_{p}(k)-\alpha} n^{\prime}$ implies $s \equiv 0\left(\bmod p^{\alpha}\right)$.

Let now suppose $C_{p}(n)<C_{p}(k)+\alpha$. Using once more Lemma 1, we shall prove that the cyclic permutation $\sigma=(1,2, \ldots, n)$ is not $\left(p^{\alpha}, k\right)$-complementing. We shall consider two cases, in each indicating an edge $e \in\left(\begin{array}{c}V_{n} \\ k\end{array}\right)$ and $s \not \equiv 0\left(\bmod p^{\alpha}\right)$ such that $\sigma^{s}(e)=e$.

Let $n^{\prime}$ and $k^{\prime}$ be such that $n=p^{C_{p}(n)} n^{\prime}$ and $k=p^{C_{p}(k)} k^{\prime}$. Note that $n^{\prime}$ and $k^{\prime}$ are integers and $k^{\prime}, n^{\prime} \not \equiv 0(\bmod p)$.

Case 1: $C_{p}(n)<C_{p}(k)$. Since $k=p^{C_{p}(n)}\left(p^{C_{p}(k)-C_{p}(n)} k^{\prime}\right)<p^{C_{p}(n)} n^{\prime}=n$ we have $p^{C_{p}(k)-C_{p}(n)} k^{\prime}<n^{\prime}$ and thus we may define

$$
e=\bigcup_{j=0}^{p^{C_{p}(n)}-1}\left\{j n^{\prime}+1, \ldots, j n^{\prime}+p^{C_{p}(k)-C_{p}(n)} k^{\prime}\right\}
$$

It is very easy to check that $|e|=k$ and $\sigma^{n^{\prime}}(e)=e$, but $n^{\prime} \not \equiv 0\left(\bmod p^{\alpha}\right)$ since $n^{\prime} \not \equiv 0(\bmod p)$.

Case 2: $C_{p}(n) \geqslant C_{p}(k)$. Since $k<n$ we have $k^{\prime}<p^{C_{p}(n)-C_{p}(k)} n^{\prime}$ and we may define

$$
e=\bigcup_{j=0}^{p^{C_{p}(k)}-1}\left\{j p^{C_{p}(n)-C_{p}(k)}+1, \ldots, j p^{C_{p}(n)-C_{p}(k)} n^{\prime}+k^{\prime}\right\}
$$

Again, $|e|=k$ and we have $\sigma^{p^{C_{p}(n)-C_{p}(k)} n^{\prime}}(e)=e$ while $p^{C_{p}(n)-C_{p}(k)} n^{\prime} \not \equiv 0\left(\bmod p^{\alpha}\right)$ (since $n^{\prime} \not \equiv 0(\bmod p)$ and $\left.C_{p}(n)-C_{p}(k)<\alpha\right)$. 
Lemma 3 Let $n, k, p, \alpha$ be positive integers such that $k<n, \alpha \geqslant 1$ and $p$ is prime. $A$ permutation $\sigma$ be of the set $V_{n}$ with orbits $O_{1}, O_{2}, \ldots, O_{m}$ is $\left(p^{\alpha}, k\right)$-complementing if and only if for every decomposition of $k$ in the form

$$
k=h_{1}+\ldots+h_{m}
$$

such that $0 \leqslant h_{j} \leqslant\left|O_{j}\right|$ for $j=1, \ldots, m$, there is an index $j_{0}, 1 \leqslant j_{0} \leqslant m$, such that $h_{j_{0}}>0$ and $C_{p}\left(O_{j_{0}}\right) \geqslant C_{p}\left(h_{j_{0}}\right)+\alpha$.

\section{Proof.}

1. Let us suppose that $\sigma$ is a permutation of $V_{n}$ with orbits $O_{1}, \ldots, O_{m}$ and $k$ is an integer $1 \leqslant k<n$, such that for any decomposition $k=h_{1}+\ldots+h_{m}$ of $k$ such that $0 \leqslant h_{j} \leqslant\left|O_{j}\right|$ for $j=1,2, \ldots, m$ there is an index $j_{0}$ with $h_{j_{0}}>0$ and $C_{p}\left(O_{j_{0}}\right) \geqslant C_{p}\left(h_{j_{0}}\right)+\alpha$. We shall apply Lemmas 1 and 2 to prove that then $\sigma$ is $\left(p^{\alpha}, k\right)$-complementing.

Let $e \in\left(\begin{array}{c}V_{n} \\ k\end{array}\right)$ and suppose that $\sigma^{s}(e)=e$ for a positive integer $s$. Denote by $e_{j}$ the set $e_{j}=O_{j} \cap e$ and by $h_{j}$ the cardinality of $e_{j}$ for $j=1,2, \ldots, m$. Let $j_{0}$ be such that $h_{j_{0}}>0$ and $C_{p}\left(O_{j_{0}}\right) \geqslant C_{p}\left(h_{j_{0}}\right)+\alpha$.

By Lemma $2, \sigma_{j_{0}}$ is a $\left(p^{\alpha}, h_{j_{0}}\right)$-complementing permutation of the complete $h_{j_{0}}$-uniform hypergraph of order $\left|O_{j_{0}}\right|$. Hence, by Lemma 1 , we have $s \equiv 0$ (mod $\left.p^{\alpha}\right)$ and, again by Lemma $2, \sigma$ is a $\left(p^{\alpha}, k\right)$-complementing of $K_{n}^{(k)}$.

2. Let now suppose that $\sigma$ is a $\left(p^{\alpha}, k\right)$-complementing permutation of $K_{n}^{(k)}$. Let $O_{1}, \ldots, O_{m}$ be the orbits of $\sigma$ and suppose that $k=h_{1}+\ldots+h_{m}$, where $0 \leqslant h_{j} \leqslant\left|O_{j}\right|$ for $j=1, \ldots, m$. Denote by $\sigma_{1}, \ldots, \sigma_{m}$ the cycles of $\sigma$ corresponding to $O_{1}, \ldots, O_{m}$, respectively. We shall prove that there is $j_{0} \in\{1,2, \ldots, m\}$ such that $h_{j_{0}}>0$ and $C_{p}\left(O_{j_{0}}\right) \geqslant C_{p}\left(h_{j_{0}}\right)+\alpha$.

Suppose, contrary to our claim, that we have $C_{p}\left(O_{j}\right)<C_{p}\left(h_{j}\right)+\alpha$ for all $j \in\{1,2, \ldots, m\}$ such that $h_{j}>0$. By Lemma 2, for every $j \in\{1,2, \ldots, m\}$ the cyclic permutation $\sigma_{j}$ is not $\left(p^{\alpha}, k_{j}\right)$-complementing permutation of the complete $k_{j}$-uniform hypergraph of order $\left|O_{j_{0}}\right|$. Hence, by Lemma 1 , for every $j \in\{1,2, \ldots, m\}$ such that $h_{j}>0$ there is a set $e_{j} \in\left(\begin{array}{l}O_{j} \\ h_{j}\end{array}\right)$ and $s_{j} \not \equiv 0\left(\bmod p^{\alpha}\right)$, such that $\sigma_{j}^{s_{j}}\left(e_{j}\right)=e_{j}$. Let $e=e_{1} \cup \ldots \cup e_{m}$. We have $|e|=k$. Denote by $l=\operatorname{lcm}\left(s_{1}, \ldots, s_{m}\right)$ (the least common multiple of $\left.s_{1}, \ldots, s_{m}\right)$. It is clear that $\sigma^{l}(e)=e$ and $l \not \equiv 0\left(\bmod p^{\alpha}\right)$. Hence, by Lemma $1, \sigma$ is not $\left(p^{\alpha}, k\right)$-comlementing, a contradiction.

\subsection{Proof of Theorem 2}

Proof of sufficiency. Let us suppose that a permutation $\sigma$ of $V_{n}$ verifies the conditions (i) and (ii) of the theorem, but it is not $\left(p^{\alpha}, k\right)$-complementing. By Lemma 3 , there is a decomposition $k=h_{1}+\ldots+h_{m}$ of $k$ such that $0 \leqslant h_{i} \leqslant\left|O_{i}\right|$ and $C_{p}\left(O_{i}\right)<C_{p}\left(h_{i}\right)+\alpha$ for every $i=1, \ldots, m$ for which $h_{i}>0$. 
Note that if, for an integer $l$ and for an index $i \in\{1, \ldots, m\}$, we have $h_{i}>0$ and $C_{p}\left(h_{i}\right) \leqslant l$, then $C_{p}\left(O_{i}\right)<C_{p}\left(h_{i}\right)+\alpha \leqslant l+\alpha$. Hence

$$
r\left(k, p^{l+1}\right) \stackrel{\left(\bmod p^{l+1}\right)}{\equiv} \sum_{i: C_{p}\left(h_{i}\right) \leqslant l} h_{i} \leqslant \sum_{i: C_{p}\left(O_{i}\right)<l+\alpha}\left|O_{i}\right|=r\left(n, p^{l+\alpha}\right)<r\left(k, p^{l+1}\right),
$$

a contradiction.

Proof of necessity. Let us suppose now that the conditions of the theorem do not hold. Then, for any $l$ such that $k_{l} \neq 0$ we have either

$$
\begin{aligned}
& \text { 1. } r\left(n, p^{l+\alpha}\right) \geqslant r\left(k, p^{l+1}\right) \text {, or } \\
& \text { 2. } r\left(n, p^{l+\alpha}\right)<r\left(k, p^{l+1}\right) \text { and } \sum_{i: C_{p}\left(O_{i}\right)<l+\alpha}\left|O_{i}\right|>r\left(n, p^{l+\alpha}\right)
\end{aligned}
$$

We shall prove that $\sigma$ is not a $\left(p^{\alpha}, k\right)$-complementing permutation of $K_{n}^{(k)}$.

We begin by proving three claims.

Claim 1 For every $l$ such that $k_{l} \neq 0$ we have

$$
\sum_{i: C_{p}\left(O_{i}\right)<l+\alpha}\left|O_{i}\right| \geqslant r\left(k, p^{l+1}\right)
$$

\section{Proof of Claim 1.}

Case 1: $r\left(n, p^{l+\alpha}\right) \geqslant r\left(k, p^{l+1}\right)$.

By the definition of $r\left(n, p^{l+\alpha}\right)$ we know that there is an integer $b$ such that $n=b p^{l+\alpha}+r\left(n, p^{l+\alpha}\right)$. Hence $\sum_{i: C_{p}\left(O_{i}\right) \geqslant l+\alpha}\left|O_{i}\right| \leqslant b p^{l+\alpha}$, and therefore

$$
\sum_{i: C_{p}\left(O_{i}\right)<l+\alpha}\left|O_{i}\right| \geqslant r\left(n, p^{l+\alpha}\right) \geqslant r\left(k, p^{l+1}\right)
$$

Case 2: $r\left(n, p^{l+\alpha}\right)<r\left(k, p^{l+1}\right)$ and $\sum_{i: C_{p}\left(O_{i}\right)<l+\alpha}\left|O_{i}\right|>r\left(n, p^{l+\alpha}\right)$. Since $\sum_{i: C_{p}\left(O_{i}\right) \geqslant l+\alpha}\left|O_{i}\right| \equiv 0\left(\bmod p^{l+\alpha}\right)$, we have

$$
\begin{gathered}
n=\sum_{i: C_{p}\left(O_{i}\right) \geqslant l+\alpha}\left|O_{i}\right|+\sum_{C_{p}\left(O_{i}\right)<l+\alpha}\left|O_{i}\right| \stackrel{\left(\bmod p^{l+\alpha}\right)}{\equiv} \\
\stackrel{\left(\bmod p^{l+\alpha}\right)}{=} \sum_{C_{p}\left(O_{i}\right)<l+\alpha}\left|O_{i}\right|>r\left(n, p^{l+\alpha}\right) \equiv n\left(\bmod p^{l+\alpha}\right)
\end{gathered}
$$

Hence there is a positive integer $d$ such that

$$
\sum_{C_{p}\left(O_{i}\right)<l+\alpha}\left|O_{i}\right|=d p^{l+\alpha}+r\left(n, p^{l+\alpha}\right) \geqslant p^{l+1}>r\left(k, p^{l+1}\right)
$$

This completes the proof of the claim.

To see that the next claim is true it is sufficient to represent $x \in \mathbf{N}$ in basis $p$. 
Claim 2 For any nonnegative integers $l, l^{\prime}, x$ and a, such that $l^{\prime} \leqslant l, x<a p^{l}, C_{p}(x) \geqslant l^{\prime}$ and $1 \leqslant a<p$ we have $x+p^{l^{\prime}} \leqslant a p^{l}$.

Claim 3 Let $u_{1}, \ldots, u_{q}$ be positive integers such that $C_{p}\left(u_{i}\right) \leqslant l+\alpha-1$ and $\sum_{i=1}^{q} u_{i} \geqslant a p^{l}$, $(0 \leqslant a<p)$. Then there exist $v_{1}, \ldots, v_{q}$ such that

(1) For every $i \in\{1, \ldots, q\} v_{i} \leqslant u_{i}$,

(2) For every $i \in\{1, \ldots, q\}$ either $C_{p}\left(u_{i}\right) \leqslant C_{p}\left(v_{i}\right)+\alpha-1$ or $v_{i}=0$,

(3) $\sum_{i=1}^{q} v_{i}=a p^{l}$.

\section{Proof of Claim 3.}

Without loss of generality we may suppose that

$$
C_{p}\left(u_{1}\right) \geqslant C_{p}\left(u_{2}\right) \geqslant \ldots \geqslant C_{p}\left(u_{q}\right)
$$

For every $i=1, \ldots, q$ denote by $l_{i}=\min \left\{C_{p}\left(u_{i}\right), l\right\}$.

The conditions (1)-(3) are satisfied by the following sequence $\left(v_{i}\right)_{i=1}^{q}$.

$$
\begin{gathered}
v_{1}=c_{1} p^{l_{1}} \text { where } c_{1}=\max \left\{c \in \mathbf{N}: c p^{l_{1}} \leqslant u_{1} \text { and } c p^{l_{1}} \leqslant a p^{l}\right\} \\
v_{2}=c_{2} p^{l_{2}} \text { where } c_{2}=\max \left\{c \in \mathbf{N}: c p^{l_{2}} \leqslant u_{2} \text { and } v_{1}+c p^{l_{2}} \leqslant a p^{l}\right\} \\
\cdots \\
v_{i}=c_{i} p^{l_{i}} \text { where } c_{i}=\max \left\{c \in \mathbf{N}: c p^{l_{i}} \leqslant u_{i} \text { and } v_{1}+\ldots+v_{i-1}+c p^{l_{i}} \leqslant a p^{l}\right\}
\end{gathered}
$$

In fact,

1. $v_{i} \leqslant u_{i}$ by the definition of $c_{i}$.

2. Since $l \geqslant C_{p}\left(u_{i}\right)-\alpha+1$ we have $C_{p}\left(v_{i}\right) \geqslant l_{i}=\min \left\{C_{p}\left(u_{i}\right), l\right\} \geqslant C_{p}\left(u_{i}\right)-\alpha+1$, whenever $v_{i} \neq 0$, thus $(2)$.

3. Suppose that the sequence $\left(v_{i}\right)_{i=1, \ldots, q}$ violates the condition (3) of the claim. Then $\sum_{i=1}^{q} v_{i}<a p^{l}$ and by consequence there is $j \in\{1, \ldots, q\}$ such that $v_{j}<u_{j}$. By Claim 2 we have $v_{j}+p^{l_{j}}=\left(c_{j}+1\right) p^{l_{j}} \leqslant u_{j}$ and $v_{1}+\ldots+\left(c_{j}+1\right) p^{l_{j}} \leqslant a p^{l}$, contrary to the choise of $c_{j}$.

The claim is proved.

We shall indicate now such a decomposition of $k$ in the form $k=h_{1}+\ldots+h_{m}$ that

(1) $h_{1}, \ldots, h_{m}$ are non negative integers,

(2) $h_{i} \leqslant\left|O_{i}\right|$ for every $i=1, \ldots, m$. 
(3) $C_{p}\left(O_{i}\right) \leqslant C_{p}\left(h_{i}\right)+\alpha-1$ or $h_{i}=0$ for every $i=1, \ldots, m$.

By Lemma 3 , this means that $\sigma$ is not $\left(p^{\alpha}, k\right)$-complementing.

Let $k=k_{l_{t}} p^{l_{t}}+k_{l_{t-1}} p^{l_{t-1}}+\ldots+k_{l_{0}} p^{l_{0}}$, where $0<k_{l_{j}}<p$ for $j=0, \ldots, t$ and $l_{0}<l_{1}<\ldots<l_{t}$, By Claim 1 we have $\sum_{i: C_{p}\left(O_{i}\right) \leqslant l_{0}+\alpha-1}\left|O_{i}\right| \geqslant k_{l_{0}} p^{l_{0}}$. Now apply Claim 3 to construct $h_{1}^{(0)}, \ldots, h_{m}^{(0)}$ such that

$\left(1_{0}\right) h_{i}^{(0)} \leqslant\left|O_{i}\right|$ for $i=1, \ldots, m$,

$\left(2_{0}\right) h_{i}^{(0)}=0$ if $C_{p}\left(O_{i}\right) \geqslant l_{0}+\alpha, i=1, \ldots, m$,

$\left(3_{0}\right) C_{p}\left(O_{i}\right) \leqslant C_{p}\left(h_{i}^{(0)}\right)+\alpha-1$ for $i$ such that $h_{i}^{(0)}>0$ and $C_{p}\left(O_{i}\right)<l_{0}+\alpha, i=1, \ldots, m$,

$\left(4_{0}\right) \sum_{i=1}^{m} h_{i}^{(0)}=k_{l_{0}} p^{l_{0}}$.

If $t=0$ set $h_{i}=h_{i}^{(0)}$ for $i=1, \ldots, m$ and the proof is finished. So we assume that $t \geqslant 1$.

Suppose we have constructed the sequences of non negative integers $\left(h_{i}^{(j)}\right)_{i=1, \ldots, m}$ for $j=0, \ldots, s-1,1 \leqslant s \leqslant t$, such that

$\left(1_{s-1}\right) h_{i}^{(0)}+h_{i}^{(1)}+\ldots+h_{i}^{(s-1)} \leqslant\left|O_{i}\right|$ for $i=1, \ldots, m$,

$\left(2_{s-1}\right) h_{i}^{(0)}+h_{i}^{(1)}+\ldots+h_{i}^{(s-1)}=0$ if $C_{p}\left(O_{i}\right) \geqslant l_{s-1}+\alpha, i=1, \ldots, m$,

$\left(3_{s-1}\right) C_{p}\left(O_{i}\right) \leqslant C_{p}\left(h_{i}^{(j)}\right)+\alpha-1$ if $h_{i}^{(j)}>0, C_{p}\left(O_{i}\right)<l_{j}+\alpha, j=0, \ldots, s-1$

$\left(4_{s-1}\right) \sum_{i=1}^{m} h_{i}^{(j)}=k_{l_{j}} p^{l_{j}}$ for $j=0, \ldots, s-1$.

We shall apply Claims 1 and 3 to construct the sequence $h_{1}^{(s)}, \ldots, h_{m}^{(s)}$ such that

$\left(1_{s}\right) h_{i}^{(0)}+h_{i}^{(1)}+\ldots+h_{i}^{(s)} \leqslant\left|O_{i}\right|$ for $i=1, \ldots, m$,

$\left(2_{s}\right) h_{i}^{(0)}+h_{i}^{(1)}+\ldots+h_{i}^{(s)}=0$ if $C_{p}\left(O_{i}\right) \geqslant l_{s}+\alpha, i=1, \ldots, m$,

$\left(3_{s}\right) C_{p}\left(O_{i}\right) \leqslant C_{p}\left(h_{i}^{(s)}\right)+\alpha-1$ whenever $C_{p}\left(O_{i}\right)<l_{s}+\alpha$ and $h_{i}^{(s)}>0, i=1, \ldots, m$,

(4s) $\sum_{i=1}^{m} h_{i}^{(s)}=k_{l_{s}} p^{l_{s}}$.

By Claim 1, we have $\sum_{i: C_{p}\left(O_{i}\right) \leqslant l_{s}+\alpha-1}\left|O_{i}\right| \geqslant r\left(k, p^{l_{s}+1}\right)=k_{l_{s}} p^{l_{s}}+k_{l_{s-1}} p^{l_{s-1}}+\ldots+k_{l_{s}} p^{l_{s}}$. Write $\lambda_{i}=\min \left\{C_{p}\left(h_{i}^{(j)}\right): h_{i}^{(j)}>0, j=1, \ldots, s-1\right\}$, for $i=1, \ldots, m$.

We have $h_{i}^{(0)}+h_{i}^{(1)}+\ldots+h_{i}^{(s-1)}=p^{\lambda_{i}} a$, where $a$ is an integer, hence

$$
C_{p}\left(O_{i}\right) \leqslant \lambda_{i}+\alpha-1 \leqslant C_{p}\left(h_{i}^{(0)}+h_{i}^{(1)}+\ldots+h_{i}^{(s-1)}\right)+\alpha-1 .
$$

Set $u_{i}=\left|O_{i}\right|-\sum_{j=0}^{s-1} h_{i}^{(j)}$ for $i=1, \ldots, m$. We have $\sum_{i=1}^{m} u_{i} \geqslant k_{l_{s}} p^{l_{s}}$ so, by Claim 3, there exist non negative integers $h_{1}^{(s)}, \ldots, h_{m}^{(s)}$ with desired properties $\left(1_{s}\right)-\left(4_{s}\right)$. 
For every $i=1, \ldots, m$ write $h_{i}=\sum_{j=0}^{t} h_{i}^{(j)}$. It is clear that $h_{i} \leqslant\left|O_{i}\right|$ for $i=1, \ldots, m$ and $\sum_{i=1}^{m} h_{i}=k$.

Repeating the argument applied above we prove easily the inequalities

$$
C_{p}\left(O_{i}\right) \leqslant C_{p}\left(h_{i}\right)+\alpha-1
$$

whenever $h_{i} \neq 0, i=1, \ldots, m$. This proves that the sequence $\left(h_{i}\right)_{i=1}^{m}$ gives the desired decomposition of $k$.

\subsection{Proof of Theorem 3}

The proof of Theorem 3 follows by Theorem 2 and the following lemma.

Lemma 4 Let $k, n, p_{1}, \ldots, p_{u}, \alpha_{1}, \ldots, \alpha_{u}$ be positive integers such that $k<n$ and $p_{1}, \ldots, p_{u}$ are primes. Write $q=p_{1}^{\alpha_{1}} \cdot \ldots \cdot p_{u}^{\alpha_{u}}$.

A permutation $\sigma$ of $V_{n}$ is $(q, k)$-complementing if and only if $\sigma$ is $\left(p_{i}^{\alpha_{i}}, k\right)$-complementing for $i=1, \ldots, u$.

Proof. By Lemma 1, a permutation $\sigma: V_{n} \rightarrow V_{n}$ is $(q, k)$-complementing if and only if for every $e \in\left(\begin{array}{c}V_{n} \\ k\end{array}\right) \sigma^{s}(e)=e$ implies $s \equiv 0(\bmod q)$. But $s \equiv 0(\bmod q)$ if and only if $s \equiv 0\left(\bmod p_{i}^{\alpha_{i}}\right)$ for every $i \in\{1, \ldots, u\}$. The lemma follows.

\section{Cyclic partitions of general complete hypergraphs}

By $\tilde{K}_{n}$ we denote the complete hypergraph on the set of vertices $V_{n}$, i.e. the hypergraph with the set of edges consisting of all non trivial subsets of $V_{n}\left(\tilde{K}_{n}=\left(V_{n} ; 2^{V_{n}}-\left\{\emptyset, V_{n}\right\}\right)\right)$. To stress the distinction between $\tilde{K}_{n}$ and $K_{n}^{(k)}$ we shall call $\tilde{K}_{n}$ the general complete hypergraph. Let $\sigma$ be a permutation of $V_{n}$. If there is a p-partition $\left\{E, \sigma(E), \ldots, \sigma^{p-1}(E)\right\}$ of $2^{V_{n}}-\left\{\emptyset, V_{n}\right\}$ then we call it cyclic $p$-partition of $\tilde{K}_{n}$ and permutation $\sigma$ is then called $p$-complementing. In [18] Zwonek proved that a cyclic 2-partition of the complete general hypergraph $\tilde{K}_{n}$ exists if and only if $n$ is a power of 2 and every 2-complementing permutation is cyclic (i.e. has exactly one orbit). Note that every partition of $\tilde{K}_{n}$ (and of $K_{n}^{(k)}$ as well) into two isomorphic parts is necessarily cyclic 2-partition.

Theorem 7 The general complete hypergraph $\tilde{K}_{n}$ has a cyclic p-partition if and only if $p$ is prime and $n$ is a power of $p(p<n)$. Moreover, every $p$-complementing permutation is cyclic.

Proof. Note first that the general complete hypergraph $\tilde{K}_{n}$ has a cyclic $p$-partition if and only if every $k$-uniform complete hypergraph $K_{n}^{(k)}$ has a cyclic $p$-partition for $1 \leqslant k \leqslant n-1$. Let us suppose first that $\tilde{K}_{n}$ has a cyclic $p$-partition and $\sigma$ is its $p$-complementing permutation.

The permutation $\sigma$ is cyclic. In fact, suppose that $\left(a_{i_{1}}, \ldots, a_{i_{k}}\right)$ is a cycle of $\sigma$, where $1 \leqslant k \leqslant n-1$. Then $\sigma\left(\left\{a_{i_{1}}, \ldots, a_{i_{k}}\right\}\right)=\left\{a_{i_{1}}, \ldots, a_{i_{k}}\right\}$, which is impossible. 
Suppose now that $p_{1}$ is a prime divisor of $p$. Let us denote $k=\frac{p}{p_{1}}$ and $e=\left\{p_{1}, 2 p_{1}, \ldots, k p_{1}\right\}$. We have $\sigma^{p_{1}}(e)=e$ hence, by Lemma $1, p_{1} \equiv 0(\bmod p)$. Since $p_{1}$ is a divisor of $p$ we obtain $p=p_{1}$.

It remains to prove that $n$ is a power of $p$. Write $\beta=\max \left\{\gamma \in \mathbf{N}: p^{\gamma} \leqslant n\right\}$. Suppose that $p^{\beta}<n$. We shall apply Theorem 2 to prove that there is no cyclic $p$-partition of $K_{n}^{\left(p^{\beta}\right)}$. Since $p^{\beta+1}>n$ we have $r\left(n, p^{\beta+1}\right)=n>r\left(p^{\beta}, p^{\beta+1}\right)=p^{\beta}$ contradicting the condition (i) in Theorem 2 (for $\alpha=1$ and $k=p^{\beta}$ ).

Let us suppose now that $p$ is prime, $n=p^{\beta}$ where $\beta$ is a positive integer. We shall prove that for any integer $k, 0<k<n$, the permutation $\sigma=(1,2, \ldots, n)$ is $(p, k)$-complementing. Let us write $k=k_{l} p^{l}+k_{l-1} p^{l-1}+\ldots+k_{o}$, where $0 \leqslant k_{i}<p$ and $k_{l} \neq 0$. We shall again apply Theorem 2 , for $\alpha=1$. In fact, note that since $r\left(k, p^{l+1}\right)=k>r\left(p^{\beta}, p^{l+1}\right)=0$ and $C_{p}(n)=\beta \geqslant l+1$ there is no orbit $O_{i}$ of $\sigma$ with $C_{p}\left(O_{i}\right)<l+1$. Hence the both conditions of Theorem 2 are verified and the proof is complete.

Acknowledgement. The authors would like to thank the anonymous referee for the thorough reading of the manuscript and helpful comments.

\section{References}

[1] L. Adamus, B. Orchel, A. Szymański, A.P. Wojda and M. Zwonek, A note on tcomplementing permutations for graphs, Information Processing Letters 110 (2009) 44-45.

[2] J.W.L. Glaisher, On the residue of a binomial coefficient with respect to a prime modulus, Quarterly Journal of Mathematics 30 (1899) 150-156.

[3] S. Gosselin, Generating self-complementary uniform hypergraphs, Discrete Math. 301 (2010) 1366-1372.

[4] S. Gosselin, Cyclically t-complementary uniform hypergraphs, to appear in the European Journal of Combinatorics.

[5] S.H. Kimball, T.R. Hatcher, J.A. Riley and L. Moser, Solution to problem E1288: Odd binomial coefficients. Amer. Math. Monthly 65 (1958) 368-369.

[6] M. Knor and P. Potočnik, A note on 2-subset-regular self-complementary 3-uniform hypergraphs, preprint.

[7] W. Kocay, Reconstructing graphs as subsumed graphs of hypergraphs, and some selfcomplementary triple systems. Graphs and Combinatorics 8 (1992) 259-276.

[8] E.E. Kummer, Über die Ergrentzungssätze zu den allgemeinen Reziprozitätsgesetzen, J. Reine Angew. Math., 44 (1852) 93-146.

[9] E. Lucas, Sur les congruences des nombres eulériens et des coefficients differentiels, Bull. Soc. Math. France 6 (1878) 49-54.

[10] P. Potočnik and M. Šajna, Regular self-comlementary uniform hypergraphs, preprint. 
[11] P. Potǒcnik and Šajna, Vertex-transitive self-complementary uniform hypergraphs, European J. Combin. 30 (2009) 327-337.

[12] P. Ribenboim, Fermat's Last Theorem for Amateurs, Springer Verlag 1999.

[13] G. Ringel, Selbstkomplementäre Graphen, Arch. Math. 14 (1963) 354-358.

[14] H. Sachs, Über selbstkomplementäre Graphen. Publ. Math. Debrecen 9 (1962) 270288.

[15] A. Szymański and A.P. Wojda, A note on k-uniform self-complementary hypergraphs of given order, Discuss. Math. Graph Theory 29 (2009) 199-202.

[16] A. Szymański and A.P. Wojda, Self-complementing permutations of k-uniform hypergraphs, Discrete Mathematics and Theoretical Computer Science 11:1 (2009) 117124.

[17] A.P. Wojda, Self-complementary hypergraphs, Discuss. Math. Graph Theory 26 (2006) 217-224.

[18] M. Zwonek, A note on self-complementary hypergraphs, Opuscula Mathematica 25/2 (2005) 351-354. 